\title{
A New Model of Thermocapillary Convection in Floating Half Zone
}

\author{
W.R. Hu, Q.S. Chen, J.H. Han, Y. A, R. Zhou \\ (Institute of Mechanics, CAS, Beijing 100080, China)
}

\begin{abstract}
A new model of thermocapillary convection in floating half zone was suggested in the present paper. The liquid bridge floats between two co-axis rods, the lower rod consists of metal with constant temperature and the upper rod consists of thermal insulating materials, where the normal gradient of temperature is nearly zero. In this case, the new model is relatively closer to simulate a half part of floating full zone in microgravity environment in comparison with the usual model of floating half zone. Basic features of the new model were studied by both numerical simulation and experiments, and the comparisons with the usual model were also discussed.

C) 1998 COSPAR. Published by Elsevier Science Ltd.

\section{Introduction}

Thermocapillary convection is a typical subject in microgravity science, and the model of floating zone has been studied extensively due to its importance in both understanding the mechanism of attractive convection and the application of materials processing in microgravity environment. The usual model of floating half zone has been studied extensively since the middle of 1970's [1] for learning the convection in a liquid bridge floating between two co-axial metal rods with same diameters and different constant temperatures. This model is beneficial for understanding the mechanism of thermocapillary convection, however, the constant temperature boundary conditions are quite different from the one of half part of a floating full zone, where the temperature at the middle heater plane should has nearly zero value of its normal gradient in the microgravity environment but does not keep constant. Therefore, a simulation model of floating half zone can be suggested, and the temperature boundary condition at upper rod of half zone may be satisfied more closely as suggested by the floating full zone condition.
\end{abstract}

A new model of floating half zone, named the simulation model, consists of a metal lower rod with constant temperature and a thermal insulating upper rod with nearly zero value of normal gradient of temperature. Due to the condition of heat flux conservation, temperature boundary condition at upper rod should be

$$
k \cdot(\nabla T)_{n}=k_{s}\left(\nabla T_{s}\right)_{n}, \quad \text { at upper boundary }
$$


where $\mathrm{k}$ and $\mathrm{T}$ are, respectively the heat conductivity and temperature, and subscript $\mathrm{s}$ and $n$ denote respectively the solid of upper rod and the normal direction of upper rod boundary. The normal gradient of temperature is nearly zero at the upper rod if heat conductivity $k$ of liquid medium is much larger than the one of upper rod. Therefore, the temperature condition at upper rod of simulation model is similar to the one at the heater plane of a floating full zone in microgravity condition, and may be constructed by selecting the materials of upper rod, which heat conductivity should be smaller than the one of liquid bridge medium. In this case, the properties of simulation model is closer to the one of half part of a florating full zone in comparison with the model of usual floating half zone. Preliminary results were given respectively by numerical simulation [2] and experiments [3].

A model of similar idea has been studied experimentally by Hirata and his colleagues since the early of 1990's [4-6] and recently by Lees [7]. A transparent upper disk of sapphire [4-6] or glass [7] is used, and the medium of experimental liquid is silicon oil. It could be seen that the heat conductivities of sapphire and glass are at least one order of magnitude larger than the one of silicon oil. The experimental results show that, the liquid temperature near the upper disk boundary is nearly constant, and has a small negative radial gradient, that is, a little higher temperature near disk center and lower temperature near free surface. Therefore, these models are relatively closer to the one of the usual model as discussed in [7], but not the model of half part of a floating full zone, which radial gradient of temperature near upper rod should be positive. It seems that the interestings of their studies are concentrated, in some sense, in the flow patterns in the horizontal cross-section of liquid bridge due to the transparence of upper rod.

The basic features of simulation model will be discussed in the present paper for the cases in microgravity condition. Numerical simulation give the temperature and velocity distributions depending on the Marangoni number and geometrical aspect ratio as shown in the next section. Experimental conditions and results for steady convection are described in section III. Onset of oscillation and the oscillatory features are summarized in section IV. The last section is conclusion.

\section{Numerical Simulation}

Numerical simulation of steady and axis-symmetric convection in simulation model of floating half zone will be discussed especially in this section by using the same numerical method as [2], where comparisons of three kind models was given. The free surface of liquid bridge is assumed to be a cylinder for simplification. The cylindrical coordinate system with origin at center of lower circular rod and symmetric line as $\mathbf{z}$ coordinate. The equations and boundary conditions for simulation model are the same as ones of usual model except the temperature boundary condition at upper rod, which 


$$
\frac{\partial T}{\partial z}=0, \quad \text { at } z=1
$$

in addition to a heating source at $r=r_{0}$ and $\mathrm{z}=1$ for simulation model, where $r_{0}$ and $\mathrm{l}$ are respectively the radius of rod and height of liquid bridge. The heating source may be applied at outer edge of upper rod, and the boundary condition may be described as follows

$$
\begin{array}{ll}
\frac{\partial T}{\partial z}=0, & \mathrm{z}=1 \text { and } 0 \leq r<r_{0}-\delta, \\
T=T_{0}+\Delta T, & \mathrm{z}=1 \text { and } r_{0}-\delta \leq r \leq r_{0},
\end{array}
$$

where $\delta$ is a small scale, and may be selected as one mesh scale of numerical calculation. In this model, the applied temperature difference is defined as difference of the heater temperature $T_{0}+\Delta T$ and the temperature at lower rod $T_{0}$.

The iso-counters of temperature and stream function are shown in the upper and lower part of Fig. 1, respectively, for simulation model (left) and usual model (right) in case of Marangoni number $\mathrm{Ma}=10000$ and geometrical aspect ratio $\mathrm{A}=0.6$. It could be seen that there is a larger temperature gradient, and then a larger driven convection in the liquid bridge near the heater for the simulation model in comparison with the usual model. There is a large and positive radial gradient of temperature at upper disk for simulation model.
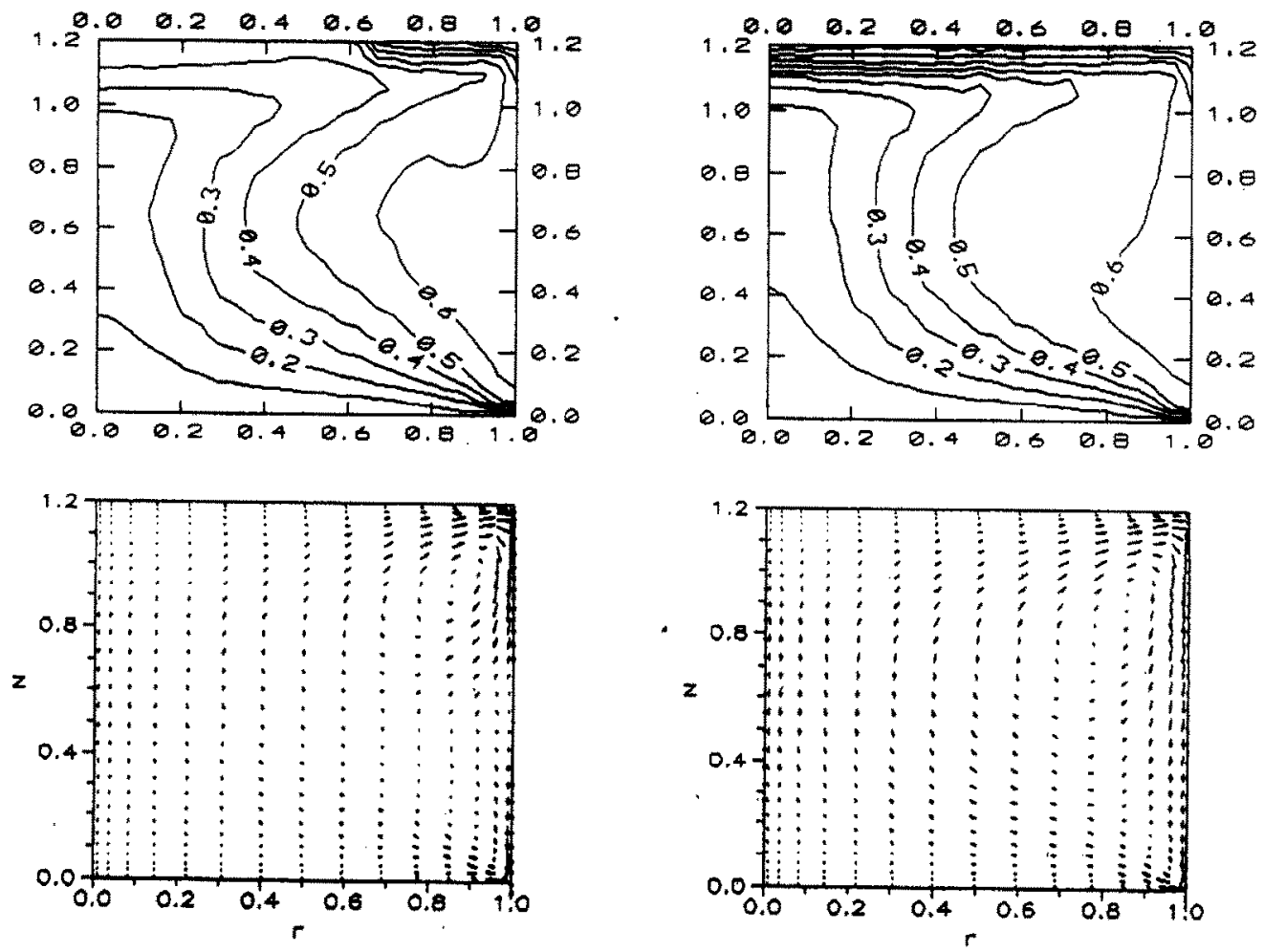

Fig. 1 Temperature profiles (upper) and flow patterns (lower) for simulation model (left) and usual model (right) in case of $\mathrm{Ma}=10000, \mathrm{~A}=0.6$. 

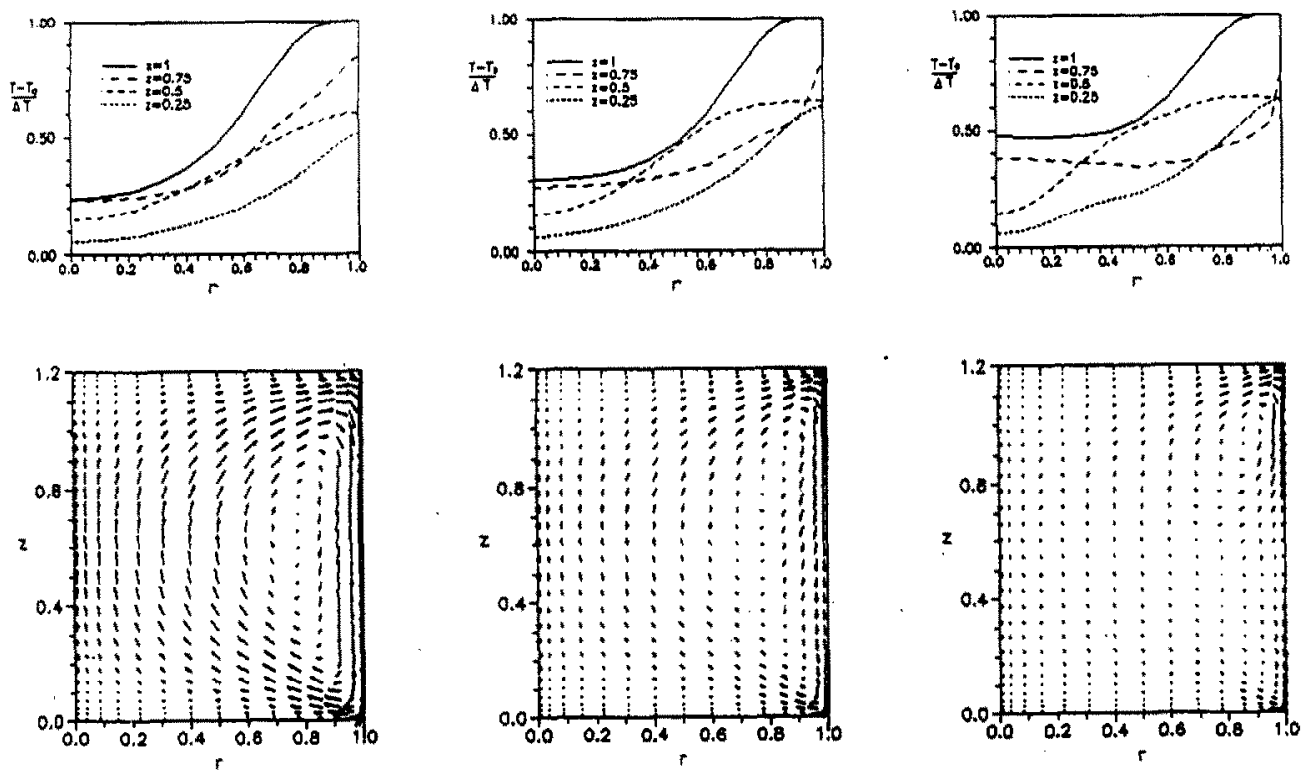

Fig. 2 Temperature profiles in horizontal cross-section (upper) and flow patterns (lower) for different Marangoni number $\mathrm{Ma}=1000$ (left), $\mathrm{Ma}=5000$ (middle) and $\mathrm{Ma}=10000$ (right)
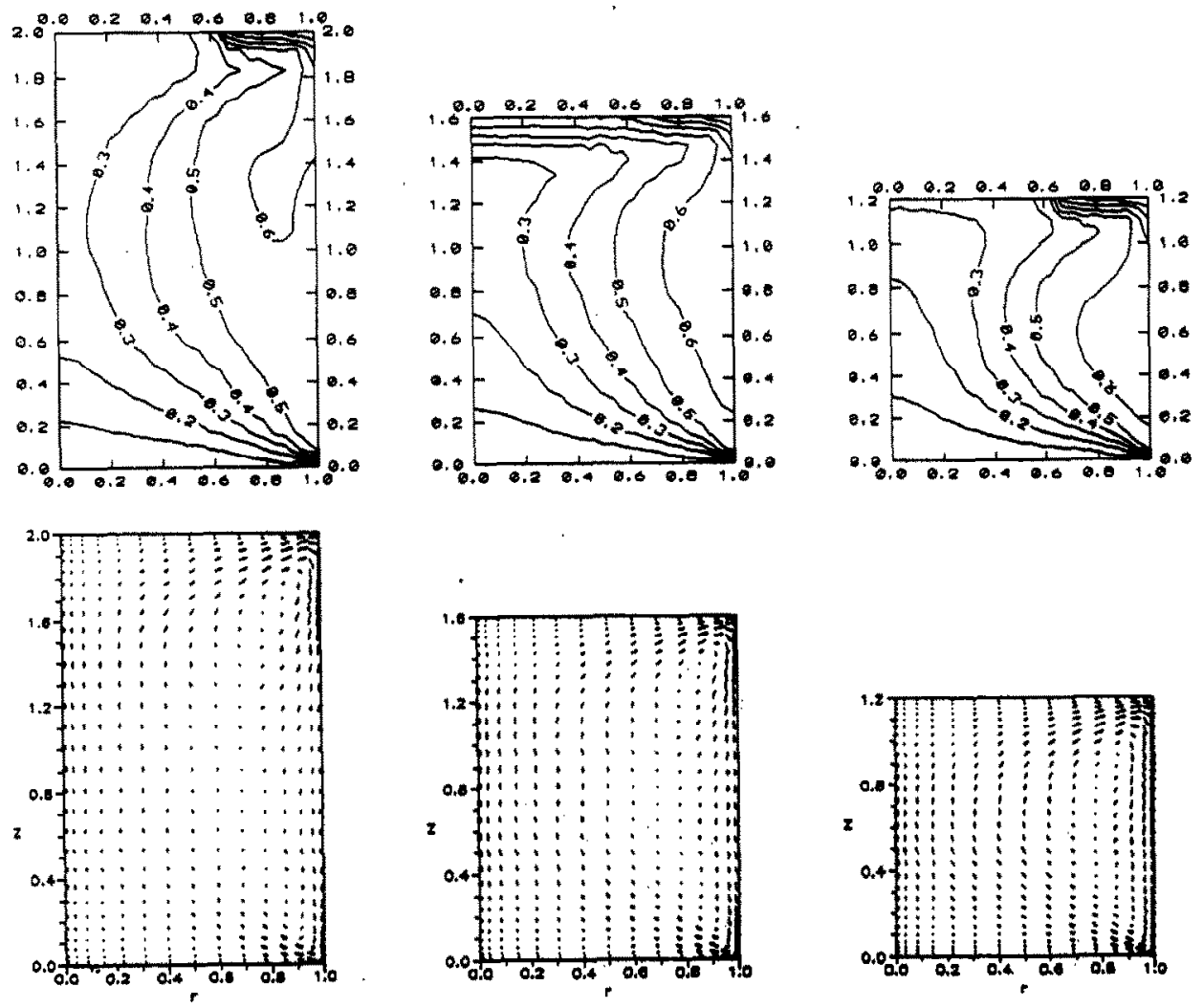

Fig. 3 The temperature distributions (upper) and flow patterns (lower) for different aspect ratio $A=1.0$ (left), $A=0.8$ (middle) and $A=0.6$ (right) in case of $\mathrm{Ma}=5000$ 
Influence of Marangoni number is shown in Fig. 2 for geometrical aspect ratio $\mathrm{A}=$ 0.6 , and the temperature profiles (upper) and stream lines (lower) are given for $\mathrm{Ma}=$ 1000 (left), 5000 (middle) and 10000 (right) respectively. The larger the Marangoni number, the stronger the convection. The influence of geometrical aspect ratio is shown in Fig. 4 for Marangoni number $\mathrm{Ma}=5000$, and temperature profiles (upper) and stream lines (lower) are given for $\mathrm{A}=1.0$ (left), $\mathrm{A}=0.8$ (middle) and $\mathrm{A}=0.6$ (right)

The results of numerical simulation show that, the basic features of simulation model is closer to the one of half part of a floating full zone in comparison with the usual model

\section{Experiments}

The $10 \mathrm{cst}$ silicon oil is used as experimental medium, and its heat conductivity is $1.34 \times 10^{4} \mathrm{erg} / \mathrm{cm} . \mathrm{sec} . \mathrm{K}$. The temperature boundary condition (2.1) for simulation model requires a materials of upper rod, which heat conductivity should be smaller than the one of silicon oil. However, the silicon oil is a material of heat insulator, and it is not easy to select a solid of upper rod, which is transparent and its coefficient of heat conductivity is smaller than the one of silicon oil. In the present experiments, the polycabonate is adopted as the upper disk, and its heat conductivity is $1.59 \times 10^{4} \mathrm{erg} / \mathrm{cm} . \mathrm{sec} . \mathrm{K}$, only a little larger than the one of silicon oil. Therefore, the temperature boundary condition (2.1) can only be approximately satisfied. It could be expect that, a large and positive gradient of temperature in the liquid bridge near upper rod may be obtained.

Same experimental facility as in [3] is used in the present paper as shown schematically in Fig. 4. The diameters of lower rod and upper disk are the same $5 \mathrm{~mm}$.

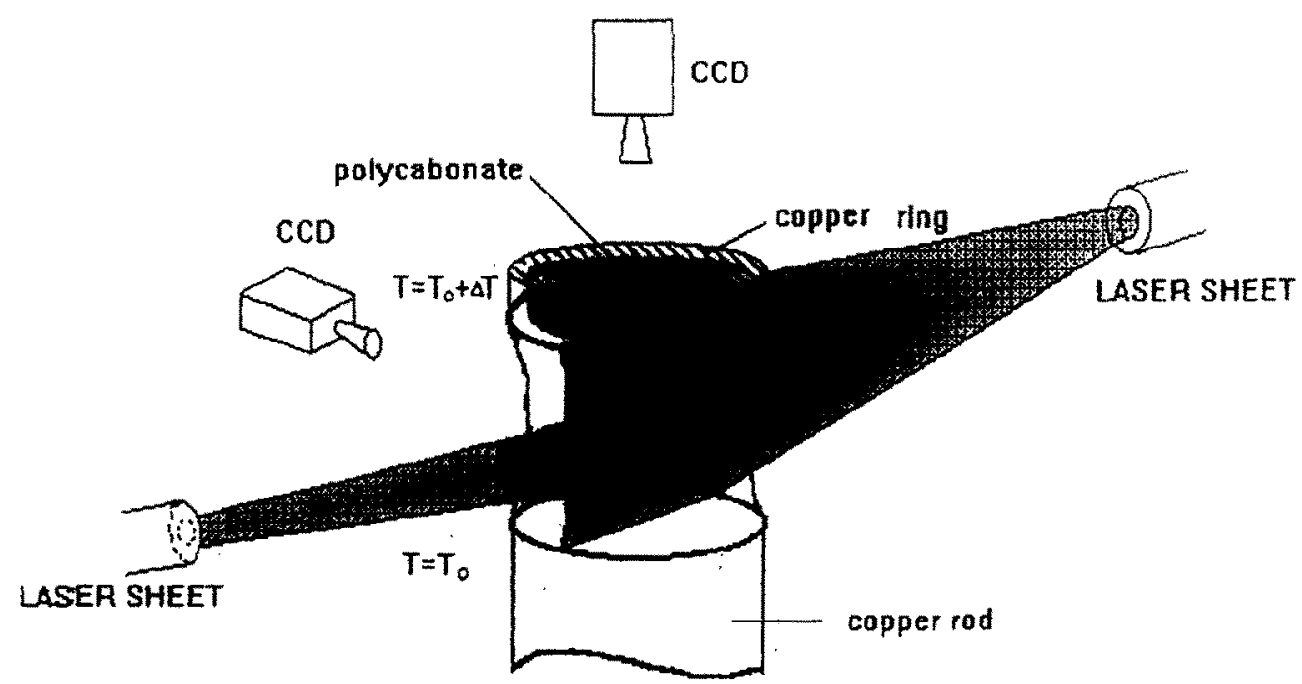

Fig. 4 Schematic diagram of experimental facility. 
There are small dynamic Bond numbers $\mathrm{Bd}=0.88$ for a geometrical aspect ratio $\mathrm{A}=$ 0.65 , and $B d=0.74$ for $A=0.55$. The small Bond number implies that, the thermocapillary effect will be more important than the effect of gravity in these parameter ranges.

The temperature distributions are measured by thermocouples and given typically in Fig. 5 for the steady convection $\Delta T=35.1^{\circ} \mathrm{C}, d_{m} / d_{0}=0.84$ and $\mathrm{A}=0.55$, where $d_{m}$ is the minimum diameter of liquid bridge. The temperature distribution of steady convection at upper rod boundary for different values of applied temperature difference are overlapped near the same profile as the one of $z / 1=1$ given in Fig. 5, and it has a larger and positive radial gradient of temperature. The temperature profile at upper disk and also the global temperature distributions in the liquid bridge agree quantitatively with the ones of numerical simulation given in Figs. 2 and 3.

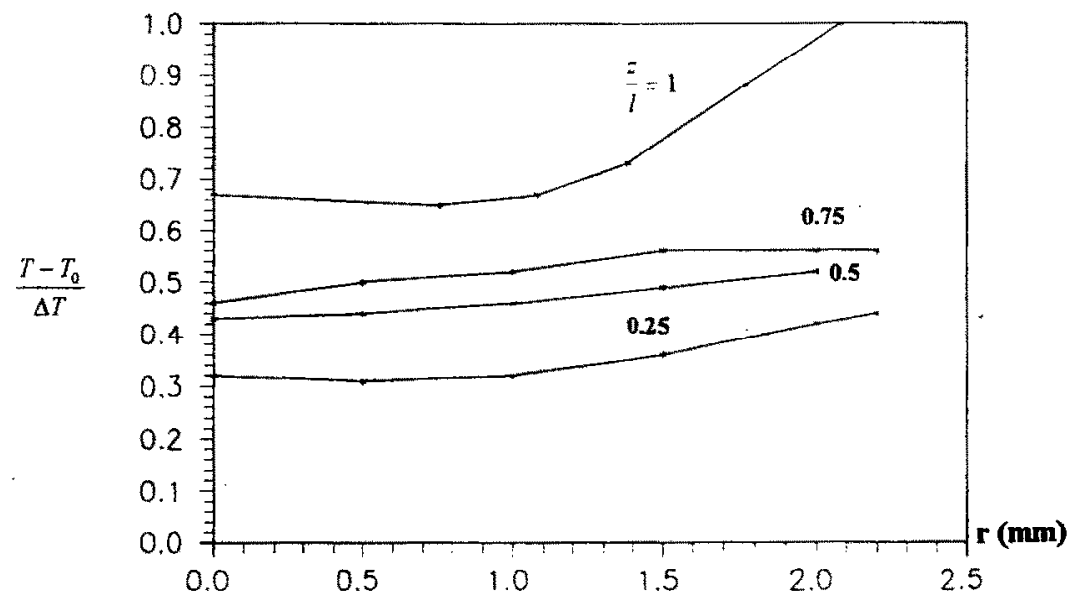

Fig. 5 Temperature distributions for case $\Delta T=35.1{ }^{\circ} \mathrm{C}, \mathrm{A}=0.55$ and $d_{m} / d_{0}=0.84$.

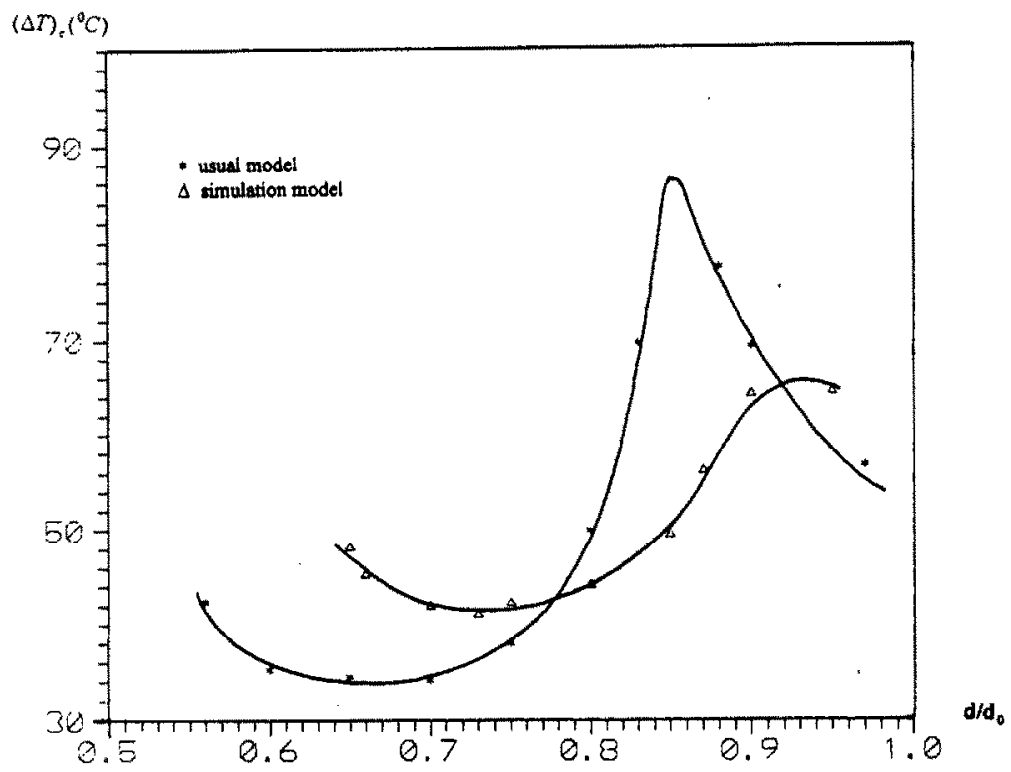

Fig. 6 Marginal curve of onset oscillation for $A=0.65$. 
The velocity fields are given by the measurement of PIV method, and the steady flow pattern may be seen typically in a vertical cross-section (upper) and in a transverse cross-section at middle height (lower) in left part of Fig. 6 for the case of $\mathrm{A}$ $=0.55$ and $d_{m} / d_{0}=0.75$. The axis-symmetric property of steady convection is clear as in the usual model of floating half zone. Due to the larger driven force near the heater, the cell center of zero velocity is closer to the free surface and heater corner in the simulation model than in the usual model.

\section{Onset of Oscillation}

The onset of oscillation for simulation model of floating half zone convection may be usually observed by the temperature oscillation and flow pattern oscillation, and also by the free surface vibration. The critical applied temperature difference depends on the volume of liquid bridge, which may be described by the ratio of minimum diameter of liquid bridge to the rod diameter, as discussed in [8] and [9] for usual model. The marginal curves of onset oscillation for both simulation model and usual

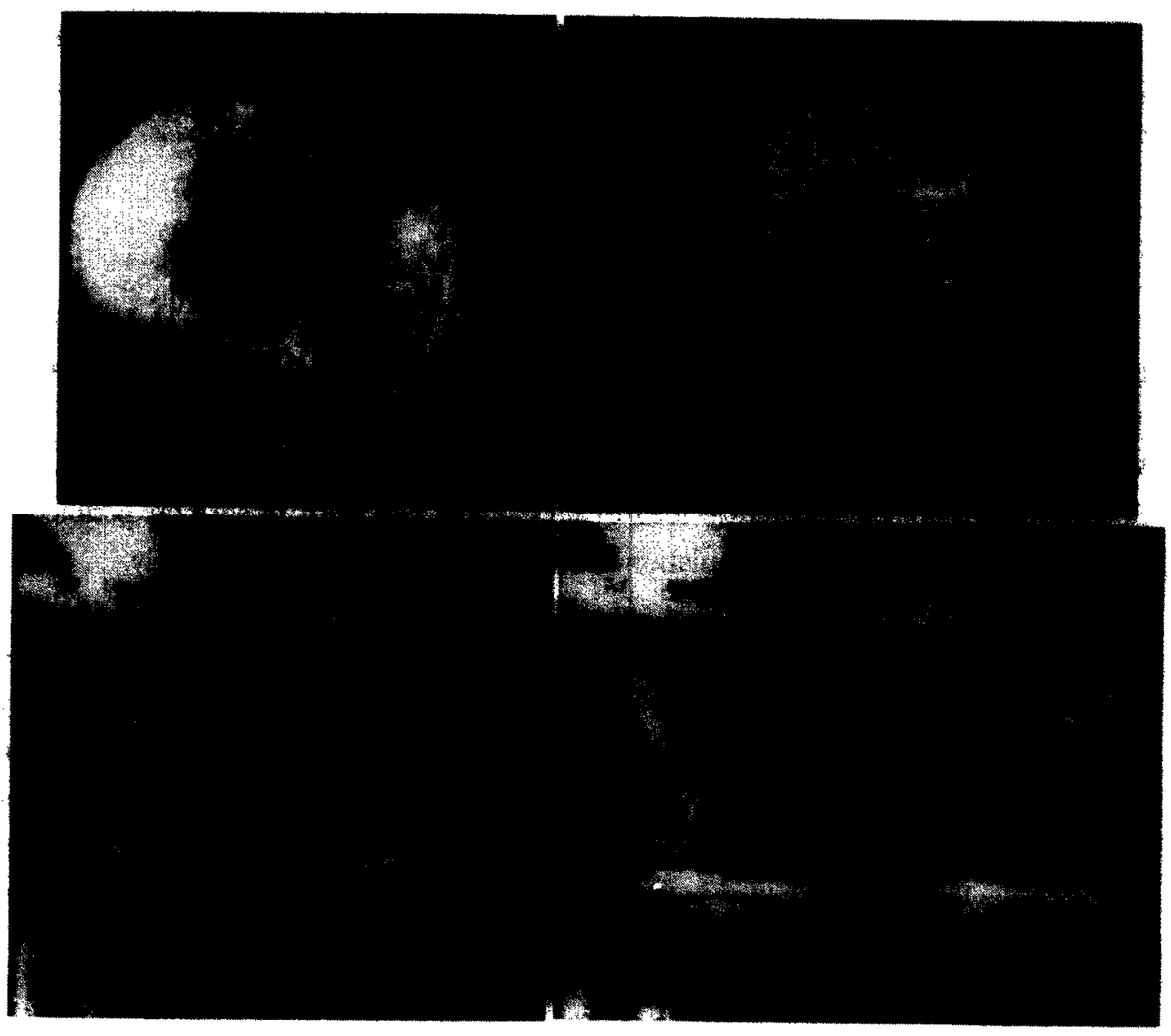

Fig. 7 Flow patterns in vertical cross-section (upper) and horizontal cross-section (lower) for steady convection (left) and oscillatory convection (right) in case $\mathrm{A}=0.55$ and $d_{m} / d_{0}=0.75$. 
model are summarized in Fig. 6 for $\mathrm{A}=0.65$. The critical Marangoni number for simulation model is smaller than the one of usual model for volume range $0.78<$ $d_{n} / d_{0}<0.91$, which range associates with most cases of importance. the critical Marangoni number for usual model is over two times than the one for simulation model near the volume $d_{m} / d_{0}=0.85$. This conclusion implies that the oscillation is easier to be excited in the simulation model than in the usual model.

Onset of oscillation accompanies with the transition of flow patterns from axissymmetric and steady convection to asymmetric and oscillatory convection. The typical flow patterns for oscillatory convection may be observed by PIV method as shown in right part of Fig. 6 for the vertical cross-section (upper) and horizontal cross-section (lower). Some rotating pattern of flow field appears in the horizontal cross-section, and it is not sure if the mass is either rotated or not rotated together with the pattern. A sequence of flow patterns in the horizontal cross-section in one period was carefully observed. A dark region in the center part of cross section rotates in a frequency of $0.77 \mathrm{~Hz}$, which is close to the frequency of temperature oscillation, and the dark region means a region with less tracers and low velocity. The mass velocity may be determined by the trajectory of tracer, and the azimuthal velocity of mass is fluctuated in a periodical form, and the fluid mass does not rotate with frequency of temperature oscillation. This conclusion is different from the one in [7]. It is important to note that the rotation of some region pattern does not mean the rotation of fluid mass.

\section{Conclusion}

Understanding the mechanism of thermocapillary convection contributes not only to the basic research on the microgravity science, but also to the application of floating zone processings in the general sense. The simulation model suggests not only a new kind of thermocapillary convection, but also a close model to simulate the half part of a floating full zone. The theoretical idea of new model was performed by experiments, and both results of numerical simulation and experiments agree quantitatively.

Furthermore, the new model is beneficial to the mechanism studies, especially the onset of oscillation. The transition process of floating half zone has been measured by inserted thermocouple. Based on the view point of fluid mechanics, velocity fluctuation is more important in comparison with temperature. Due to the transparency of upper rod, velocity field may be observed in three dimension, and complex patterns associating with the oscillatory convection have been discovered. A lot of studies should be completed in the future.

\section{References}

1. Chang, C.E., Wilcox, W.R., J. Crystal Growth, 28 (1975) 8

2. Chen, Q.S., Hu, W.R., Numerical investigation on a simulation model of floating zone convection, Int. J. Heat and Mass Transfer, in printing 
3. Han, J.H., A. Y., Zhou, R., Hu, W.R., Investigation on a simulation model of floating zone convection, II. Experiment, Int. J. Heat and Mass transfer, submitted

4. Hirata, A., Nishizawa, S., Sakurai, M., J. Japan Society Microgravity Application, 11 (1992) 189

5. Hirata, A., Nishizawa, S., Noguchi, M., Sakurai, M., Yasuhiro, M., Imaishi, N., J. Chem. Eng. Japan, 27 (1994) 65

6. Sakuria, M., Marangoni convection in a liquid bridge under normal and microgravity conditions, Ph.D. Thesis, Department of Chemical Eng., Waseda University, 1996

7. Lee, J., Lee, D.J., Lee, J.H., J. Crystal Growth, 152 (1995) 341

8. Hu, W.R., Shu, J.Z., Zhou, R., J. Crystal Growth, 142 (1994) 379

9. Tang, Z.M., Hu, W.R., J. Crystal Growth, 142 (1994) 385 